\title{
Are you using the third definition to diagnose sepsis in clinic? - A survey among Chinese intensivists
}

\author{
Li-Hua Dong ${ }^{1}$, Qi-Yu Zhang ${ }^{2}$, Ning-Ning Di ${ }^{3}$, Qiu-Li Xue ${ }^{1}$, Ya-Jie Liu ${ }^{1}$ \\ ${ }^{1}$ Department of Intensive Care Unit, The First Hospital of Jilin University, Changchun, China; ${ }^{2}$ Department of Computed Tomography, \\ ${ }^{3}$ Department of Intensive Care Unit, General Hospital of Jilin Oil Field, Songyuan, China \\ Contributions: (I) Conception and design: LH Dong; (II) Administrative support: LH Dong, QY Zhang; (III) Provision of study materials or patients: \\ All authors; (IV) Collection and assembly of data: NN Di, QL Xue, YJ Liu; (V) Data analysis and interpretation: LH Dong, QY Zhang, NN Di; (VI) \\ Manuscript writing: All authors; (VII) Final approval of manuscript: All authors. \\ Correspondence to: Li-Hua Dong. Department of Intensive Care Unit, The First Hospital of Jilin University, 71 Xinmin Street, Changchun 130021, \\ China. Email: dongleehua@163.com.
}

Background: Since sepsis-3 definition is more accurate and sensitive than previous sepsis definition, implementation the newest diagnosis criteria could definitely bring more benefit to sepsis patients. This study was done to identify the level of current intensivists' knowledge regarding the third international consensus definitions for sepsis and septic shock and its implementation for the diagnosis of sepsis among Chinese adult intensive care units (ICUs).

Methods: A nationwide survey amongst critical care physicians was designed. The questionnaires measured the understanding and the frequency of diagnosis of sepsis and septic shock according to the third international consensus definitions for sepsis and septic shock. One thousand random physician members of Chinese Society of Critical Care Medicine were involved in the survey. A 5-point Likert scale (totally understand, partially understand, understand, hardly understand, do not understand) was used to elicit answers about the degree of understanding the sepsis-3 definition. The other 5 -point Likert scale (always, often, sometimes, rarely, never) was used to elicit answers about the frequency of diagnosing sepsis according to the sepsis-3 definition.

Results: There were 59 (16.1\%) intensivists who could completely understand the sepsis-3 definition. Less practiced intensivists could understand the sepsis-3 definition better than more practiced intensivists $(\mathrm{P}<0.001)$. Intensivists from university teaching hospitals understand the sepsis-3 definition better than the intensivists from the community hospitals $(\mathrm{P}<0.001)$. Intensivists from small-sized ICUs understand the sepsis-3 definition better than intensivists from big-sized ICUs $(\mathrm{P}<0.001)$. There were 60 (16.4\%) intensivists who always diagnose sepsis according to the sepsis-3 definition since sepsis-3 published. Less practiced intensivists prefer using the sepsis-3 definition to diagnose sepsis compared with more practiced intensivists $(\mathrm{P}<0.001)$. Intensivists from university teaching hospitals prefer using the sepsis-3 definition to diagnose sepsis compared with the intensivists from the community hospitals $(\mathrm{P}<0.001)$. Intensivists from small-sized ICUs prefer adapting sepsis-3 definition to diagnosis sepsis compared with intensivists from big-sized ICUs $(\mathrm{P}<0.001)$.

Conclusions: In current China, the sepsis-3 definition is well understood and accepted by intensivists.

Keywords: Sepsis definition; diagnosis; survey; intensive care unit (ICU)

Submitted Feb 14, 2020. Accepted for publication Jul 09, 2020.

doi: $10.21037 / \mathrm{apm}-20-412$

View this article at: http://dx.doi.org/10.21037/apm-20-412

^ ORCID: 0000-0002-8861-9056. 


\section{Introduction}

Sepsis is frequently diagnosed in intensive care units (ICUs). Although sepsis has attracted the attention of physicians and researchers because it is the leading cause of mortality and a major health burden world-widely, the definition remains debatable (1-10). The third international consensus Task Force convented by the European Society of Intensive Care Medicine and the society of Critical Care Medicine redefined sepsis as "life-threatening organ dysfunction due to a dysregulated host response to infection" $(1,11,12)$. According to the sepsis-3 definition, clinical criteria of sepsis are suspected or documented infection and an acute increase of $\geq 2$ SOFA points (a proxy for organ dysfunction); septic shock can be defined as sepsis with persisting hypotension, which requiring vasopressor therapy to elevate MAP, and lactate $>2 \mathrm{mmol} / \mathrm{L}(18 \mathrm{mg} / \mathrm{dL})$, despite adequate fluid resuscitation (12). Thus, it revised and validated the new clinical criteria for sepsis (sepsis-3). Despite the sepsis-3 definition has been published for more than 3 years, the 1991 and 2001 consensus terminology are still in use in current clinical and research work among intensivists all over the world $(13,14)$. Studies have investigated the first-hour basic care tasks of sepsis, the 6 -hour resuscitation bundle or the $24 \mathrm{~h}$ resuscitation bundle implementation $(15,16)$. Early diagnosis is always the key for good prognosis for sepsis patients. Since the sepsis-3 definition is more accurate and sensitive than the previous sepsis definition, implementation the newest diagnosis criteria could definitely bring more benefit to sepsis patients. No studies have investigated the sepsis-3 definition implementation among intensivists in China. This study was done to determine the level of current intensivists' knowledge regarding the third international consensus definitions for sepsis and septic shock and its implementation for the diagnosis of sepsis and septic shock among Chinese adult intensive care unit (ICU).

We present the following article in accordance with the SURGE report checklist (available at http://dx.doi. org/10.21037/apm-20-412).

\section{Methods}

\section{Study population and definitions}

The pilot survey was mailed to 1,000 physician members of Chinese Society of Critical Care Medicine (CSCCM) practicing within China, who was were randomly selected from the CSCCM database in April 2018. Standardized survey procedures were used throughout the study. Intensivists received individualized cover letters describing the study and self-addressed stamped envelopes for return of the completed survey via first class mail. A facsimile number was also provided for those who wished to return the survey by facsimile. A reminder mail was sent 3 weeks after the initial mailing. The study was granted approval with exempt status by Jilin University Committee on Human Research.

The survey was composed of 5 questions. Clinically relevant information included years of critical care practice, type of hospital, which included university teaching hospital or community hospital, No. of ICU beds within institution, the degree of understanding the sepsis- 3 definition, and the frequency of diagnosing sepsis according to sepsis-3 definition since sepsis-3 published. A 5-point Likert scale (totally understand, partially understand, understand, hardly understand, do not understand) was used to elicit answers about the degree of understanding the sepsis-3 definition. The other 5-point Likert scale (always, often, sometimes, rarely, never) was used to elicit answers about the frequency of diagnosing sepsis according to sepsis-3 definition.

\section{Survey validation}

Surveys of the third international consensus definitions for sepsis and septic shock and the diagnosis of sepsis and septic shock were distributed to randomly selected participants in May, 2018. One thousand physician members of the Chinese Society of Critical Care Medicine (CSCCM) were involved in the survey. Intensivist received a survey mail and a reminder mobile text message at the same time. A second mail was sent 3 weeks after the initial mail. The questionnaires were mailed to 50 adult ICUs from June to September in 2018.

\section{Statistical analysis}

Surveys results were coded and entered into a database for statistical analysis (SPSS software, version 17.0, Chicago, IL, USA). Descriptive statistics were used to compare differences in intensives demographic variables to survey responses. The chi-squared methodology was used to analyze the significance of observed differences between nominal variables in relation to survey responses. Statistical significance was defined as $\mathrm{P}<0.05$. 


\section{Results}

The survey achieved 519 responses of 1,000 (response rate was $51.9 \%$ ). Among the 519 responses, 153 were not actually practicing in ICU and were excluded from further analysis, 366 responses were usable surveys (70.5\%). Among the intensivists, $63.7 \%$ of respondents have worked in clinical practice for at least 6 years. Both university and community hospitals were adequately represented. Among the surveys, $75.9 \%$ of replying participants were from middle-sized ICU, with 10-40 beds within institution (Table 1).

Relevant data pertaining to the degree of understanding the sepsis-3 definition in Chinese intensivists is included in Table 2. Altogether there were 59 (16.1\%) intensivists could completely understand the sepsis-3 definition. There were $16(4.4 \%)$ intensivists do not understand the sepsis-3 definition. Regarding years of critical care practice, $53.9 \%$ of the intensivists with more than 12 years of critical care practice and $61.1 \%$ of the intensivists with $6-12$ years of critical care practice understand the sepsis-3 definition. While, $87.2 \%$ of the intensivists with less than 3 years of critical care practice totally understand or understand the sepsis-3 definition. Regarding types of hospital, intensivists from university teaching hospitals understand the sepsis-3 definition (53.9\% understand, $17.4 \%$ totally understand) better $(\mathrm{P}<0.001)$ than the intensivists from community hospitals (33.3\% understand, $13.0 \%$ totally understand). Regarding to the number of ICU beds within institution, the intensivists from small-sized ICUs (less than 10 beds) understand the sepsis-3 definition (45.5\% totally understand, $27.3 \%$ understand) better $(\mathrm{P}<0.001)$ than the intensivists from big-sized (more than 50 beds) ICUs $(50.0 \%$ partially understand, $20.0 \%$ do not understand). Table 2 suggests that less practiced intensivists could understand the sepsis-3 definition better than more practiced intensivists $(\mathrm{P}<0.001)$. Intensivists from university teaching hospitals understand the sepsis-3 definition better than the intensivists from the community hospitals $(\mathrm{P}<0.001)$. Intensivists from small-sized ICUs understand the sepsis-3 definition better than intensivists from big-sized ICU $(\mathrm{P}<0.001)$.

Relevant data pertaining to the frequency of diagnosing sepsis according to the sepsis-3 definition since sepsis-3 published is included in Table 3. There were 60 (16.4\%) intensivists who always diagnosis sepsis according to the sepsis-3 definition since sepsis-3 published, but 21 (5.7\%) intensivists never diagnosis sepsis according to the sepsis-3 definition. Among the intensivists with more than 12 years
Table 1 Critical care physician demographics

\begin{tabular}{lc}
\hline Demographic question & No. of respondent $(\%)^{a}$ \\
\hline Years of critical care practice & 366 \\
$>12$ & $102(27.9)$ \\
$6-12$ & $98(26.8)$ \\
$3-5$ & $35(9.6)$ \\
$<3$ & \\
Type of hospital & $258(70.5)$ \\
University teaching & $108(29.5)$ \\
Community & \\
No. of ICU beds within institution & $22(6.0)$ \\
$<10$ & $88(24.0)$ \\
$10-20$ & $103(28.1)$ \\
$21-30$ & $87(23.8)$ \\
$31-40$ & $34(9.3)$ \\
$41-50$ & $32(8.7)$ \\
$>50$ & \\
\hline
\end{tabular}

ICU, intensive care unit. ${ }^{a}$ Percent data based on the number of respondents to each question.

of critical practice, $73.6 \%$ intensivists often or always diagnosis sepsis according to the sepsis-3 definition. Among the intensivists with 6-12 years of critical care practice, $65.4 \%$ of the intensivists often or always diagnosis sepsis according to sepsis- 3 definition. But $40 \%$ of the intensivists with less than 3 years of critical care practice often or always diagnosis sepsis according to sepsis-3 definition. Regarding types of hospital, intensivists from university teaching hospitals diagnosis sepsis according to the sepsis-3 definition (18.6\% always vs. $50.0 \%$ often) more frequently $(\mathrm{P}<0.001)$ than the intensivists from community hospitals (11.1\% always vs. $1.9 \%$ often). Regarding to the number of ICU beds within institution, the intensivists from smallsized ICUs (less than 10 beds) diagnosis sepsis according to the sepsis-3 definition (9.1\% always vs. $27.3 \%$ understand) more frequently $(\mathrm{P}<0.001)$ than the intensivists from bigsized (more than 50 beds) ICUs (3.1\% always vs. $25.0 \%$ often). Table 3 suggests that less practiced intensivists prefer using the sepsis-3 definition to diagnosis sepsis compared with more practiced intensivists $(\mathrm{P}<0.001)$. Intensivists from the university teaching hospitals prefer using sepsis-3 definition to diagnosis sepsis compared with the intensivists from the community hospitals $(\mathrm{P}<0.001)$. Intensivists 
Table 2 The degree of understanding the sepsis-3 definition in Chinese intensivists

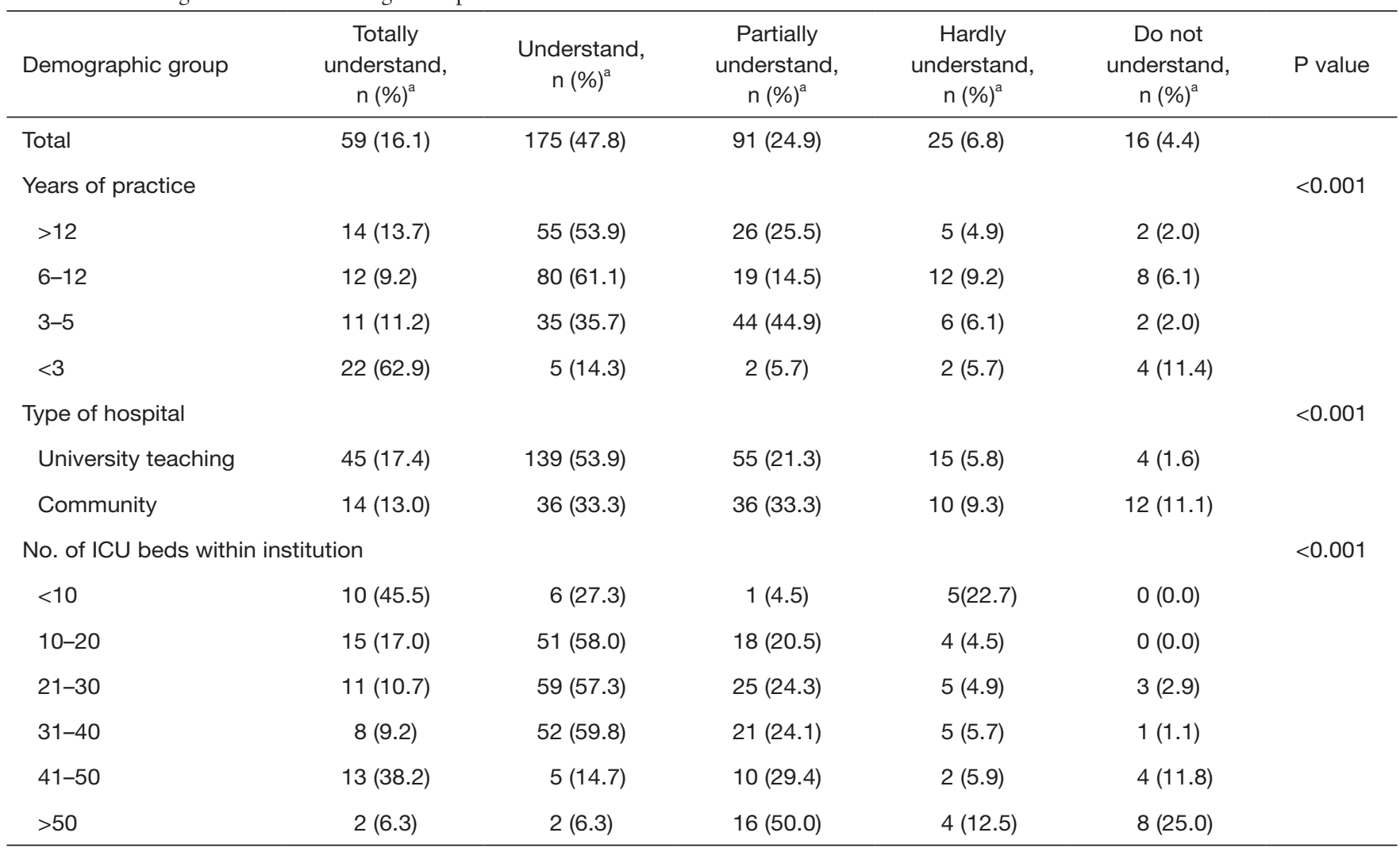

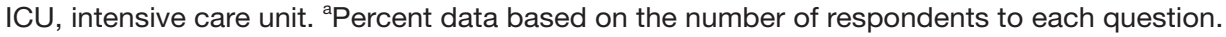

from small-sized ICUs prefer adapt sepsis-3 definition to diagnosis sepsis compared with intensivists from big-sized ICUs $(\mathrm{P}<0.001)$.

\section{Discussion}

The third international consensus Task Force convened by the European Society of Intensive Care Medicine and the Society of Critical Care Medicine redefined sepsis as "lifethreatening organ dysfunction due to a dysregulated host response to infection" $(1,11,12)$. Sepsis-3 definition revised and validated the new clinical criteria for sepsis. Though sepsis-3 definition is still in debate as the criteria for sepsis (17), the sepsis-3 definition is currently the most accurate and sensitive criteria for sepsis diagnosis $(13,14)$. This study was the first survey to determine the level of current intensivists' knowledge regarding the sepsis-3 definition and its implementation for the diagnosis and management of sepsis and septic shock among Chinese adult ICUs.

The survey suggests that in current China, the majority of intensivists understand the sepsis-3 definition, and diagnosis sepsis according to the sepsis- 3 definition. Since the morbidity and mortality of sepsis in China is relatively high (18-21), Chinese physicians have always been taking emphasize on the diagnosis and treatment of sepsis. In 2007, Chinese Society of Critical Care Medicine have published "Guidelines for emergency management of sepsis/septic shock in China", and updated in 2014 and 2018 (22). The "Guidelines for emergency management of sepsis/septic shock in China of 2018" is almost consistent with the sepsis-3 definition. Since the sepsis-3 definition is more accurate and sensitive than previous sepsis definition, and early diagnosis is always the key for good prognosis for sepsis patients, it is possible to anticipate that the morbidity of sepsis is increasing and the mortality of sepsis is decreasing in current China.

This survey also suggests the following three characters regarding the implementation of the sepsis-3 definition in current China. Firstly, less practiced intensivists understand the sepsis-3 definition better than more practiced intensivists. The possible reason is the more 
Table 3 The frequency of diagnosing sepsis according to sepsis- 3 definition since sepsis- 3 published

\begin{tabular}{|c|c|c|c|c|c|c|}
\hline Demographic group & Always, n (\%) ${ }^{a}$ & Often, n (\%) ${ }^{a}$ & $\begin{array}{c}\text { Sometimes, } \\
n(\%)^{\mathrm{a}}\end{array}$ & Rarely, n (\%) ${ }^{a}$ & Never, n (\%) & $P$ value \\
\hline Total & $60(16.4)$ & $131(35.8)$ & $111(30.3)$ & $43(11.7)$ & $21(5.7)$ & \\
\hline$>12$ & $33(32.4)$ & $42(41.2)$ & $22(21.6)$ & $3(2.9)$ & $2(2.0)$ & \\
\hline$<3$ & $10(28.6)$ & $4(11.4)$ & $11(31.4)$ & 8 (22.9) & $2(5.7)$ & \\
\hline \multicolumn{6}{|l|}{ Type of hospital } & $<0.001$ \\
\hline University teaching & $48(18.6)$ & $129(50.0)$ & $59(22.9)$ & $10(3.9)$ & $12(4.7)$ & \\
\hline $10-20$ & $35(39.8)$ & $22(25.0)$ & $25(28.4)$ & $4(4.5)$ & $2(2.3)$ & \\
\hline $21-30$ & $8(7.8)$ & $59(57.3)$ & $26(25.2)$ & $6(5.8)$ & $4(3.9)$ & \\
\hline $31-40$ & $12(13.8)$ & $30(34.5)$ & $37(42.5)$ & $5(5.7)$ & $3(3.4)$ & \\
\hline $41-50$ & $2(5.8)$ & $6(17.6)$ & $12(35.3)$ & $10(29.4)$ & $4(11.8)$ & \\
\hline$>50$ & $1(3.1)$ & $8(25.0)$ & $6(18.8)$ & $14(3.1)$ & $3(9.4)$ & \\
\hline
\end{tabular}

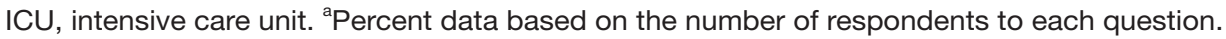

practiced intensivists get used to diagnosis sepsis according the first or second definition of sepsis. It is very common that a practiced physician makes diagnosis according to his practice, instead of the documented criteria. But the definition of sepsis is changing with people's acknowledgement about the pathological mechanism of sepsis. Secondly, intensivists from the university teaching hospitals understand the sepsis- 3 definition better than the intensivists from the community hospitals. The reason is there are more chances to attend academic conferences for intensivists who work in the university teaching hospitals. Thirdly, intensivists from small-sized ICU understand the sepsis-3 definition better than intensivists from bigsize ICU. In China, the size of ICU is different between different types of hospitals. In very advanced hospital, the beds number is usually $8-20$ beds. The big-sized ICU does not mean an advanced ICU. So, this suggests that the intensivists in more advanced ICU master the definition of sepsis-3 better, and diagnosis sepsis according to the more accurate and sensitive criteria.

This study was not without limitations and included only the CSCCM physician members. The survey achieved 519 responses of 1,000. Among the 519 responses, 153 were not actually practicing in ICUs and were excluded from further analysis, 366 responses were usable surveys $(70.5 \%)$. This is the specific population we intended to survey, the sample is small, and the response rate $(51.9 \%)$ is not high. The variability in response may be related to those mentioned factors. This investigation has led to further questioning of current mortality of sepsis in China using the sepsis-3 definition to diagnosis sepsis. In the absence of large trials, Epidemiology of the sepsis 3.0 guideline implementation in Chinese intensivists from a multidisciplinary critical care viewpoint is necessary in future.

\section{Conclusions}

In current China, the sepsis-3 definition is well understood and applied in clinical practice by intensivists. However, practice periods, hospital types and ICU size affect the implementation of the sepsis 3.0 guideline. Sepsis-3 definition are implemented better among intensivists with less practiced, from small-sized ICUs, and from university teaching hospitals. 


\section{Acknowledgments}

Funding: The experiment was supported by Natural Science Fund of Jilin Province Science and Technology Department (20180101340JC) and Scientific and Technological Ability Promotion Found of Health Commission of Jilin Province.

\section{Footnote}

Reporting Checklist: The authors have completed the SURGE Reporting checklist. Available at http://dx.doi. org/10.21037/apm-20-412

Data Sharing Statement: Available at http://dx.doi. org/10.21037/apm-20-412

Peer Review file: Available at http://dx.doi.org/10.21037/ apm-20-412

Conflicts of Interest: All authors have completed the ICMJE uniform disclosure form (available at http://dx.doi. org/10.21037/apm-20-412). The authors have no conflicts of interest to declare.

Ethical Statement: The authors are accountable for all aspects of the work in ensuring that questions related to the accuracy or integrity of any part of the work are appropriately investigated and resolved. The experiment is not involved in the animal experiments or human studies, so Ethical Approval and Informed consent is not applicable in this study.

Open Access Statement: This is an Open Access article distributed in accordance with the Creative Commons Attribution-NonCommercial-NoDerivs 4.0 International License (CC BY-NC-ND 4.0), which permits the noncommercial replication and distribution of the article with the strict proviso that no changes or edits are made and the original work is properly cited (including links to both the formal publication through the relevant DOI and the license). See: https://creativecommons.org/licenses/by-nc-nd/4.0/.

\section{References}

1. Singer M, Deutschman CS, Seymour CW, et al. The third international consensus definitions for sepsis and septic shock (sepsis-3). JAMA 2016;315:801-10.

2. Dias FS. Sepsis definitions. Rev Bras Ter Intensiva
2017;29:520-1.

3. Marshall JC. Sepsis Definitions: A Work in Progress. Crit Care Clin 2018;34:1-14.

4. Kingwell K. Sepsis: stalking a new target. Nat Rev Drug Discov 2017;16:825.

5. Wiersinga WJ, van Zanten ARH, Kaasjager KA, et al. Sepsis: new insights, new definition. Ned Tijdschr Geneeskd 2017;161:D1551.

6. Taeb AM, Hooper MH, Marik PE. Sepsis: Current Definition, Pathophysiology, Diagnosis, and Management. Nutr Clin Pract 2017;32:296-308.

7. Tusgul S, Carron PN, Yersin B, et al. Low sensitivity of qSOFA, SIRS criteria and sepsis definition to identify infected patients at risk of complication in the prehospital setting and at the emergency department triage. Scand J Trauma Resusc Emerg Med 2017;25:108.

8. Verdonk F, Blet A, Mebazaa A. The new sepsis definition: limitations and contribution to research and diagnosis of sepsis. Curr Opin Anaesthesiol 2017;30:200-4.

9. Vincent JL, Martin GS, Levy MM. qSOFA does not replace SIRS in the definition of sepsis. Crit Care 2016;20:210.

10. Wynn JL, Polin RA. Progress in the management of neonatal sepsis: the importance of a consensus definition. Pediatr Res 2018;83:13-5.

11. Shankar-Hari M, Phillips GS, Levy ML, et al. Developing a new definition and assessing new clinical criteria for septic shock: for the third international consensus definitions for sepsis and septic shock (sepsis-3). JAMA 2016;315:775-87.

12. Seymour CW, Liu VX, Iwashyna TJ, et al. Assessment of clinical criteria for sepsis: for the third international consensus definitions for sepsis and septic shock (sepsis-3). JAMA 2016;315:762-74.

13. American college of chest physicians/society of critical care medicine consensus conference: definitions for sepsis and organ failure and guidelines for the use of innovative therapies in sepsis. Crit Care Med 1992;20:864-74.

14. Levy MM, Fink MP, Marshall JC, et al. 2001 SCCM/ ESICM/ACCP/ATS/SIS International sepsis definitions conference. Intensive Care Med 2003;29:530-8.

15. Qian J, Wang Y, Zhang Y, et al. A Survey of the firsthour basic care tasks of severe sepsis and septic shock in pediatric patients and an evaluation of medical simulation on improving the Compliance of the tasks. J Emerg Med 2016;50:239-45.

16. Girardis M, Rinaldi L, Donno L, et al. Effects on management and outcome of severe sepsis and septic 
shock patients admitted to the intensive care unit after implementation of a sepsis program: a pilot study. Crit Care 2009; 13:R143.

17. Donnelly JP, Safford MM, Shapiro NI, et al. Application of the Third International Consensus Definitions for Sepsis (Sepsis-3) Classification: a retrospective populationbased cohort study. Lancet Infect Dis 2017;17:661-70.

18. Weng L, Zeng XY, Yin P, et al. Sepsis-related mortality in China: a descriptive analysis. Intensive Care Med,2018;44:1071-80.

19. Liao X, Du B, Lu M, et al. Current epidemiology of sepsis in mainland China. Ann Transl Med 2016;4:324.

20. Zhou J, Qian C, Zhao M, et al. Epidemiology and outcome of severe sepsis and septic shock in intensive care units in mainland China. PLoS One 2014;9:e107181.

21. Cheng B, Xie G, Yao S, et al. Epidemiology of severe sepsis in critically ill surgical patients in ten university hospitals in China. Crit Care Med 2007;35:2538-46.

22. Emergency physicians branch of Chinese medical doctors association. Guidelines for emergency management of sepsis/septic shock in China. Chin J Crit Care 2018;1:741-54.

Cite this article as: Dong LH, Zhang QY, Di NN, Xue QL, Liu YJ. Are you using the third definition to diagnose sepsis in clinic?-A survey among Chinese intensivists. Ann Palliat Med 2020;9(5):2926-2932. doi: 10.21037/apm-20-412 